\title{
Band Gap of Strained Graphene Nanoribbons
}

\author{
Yang Lu and Jing Guo ( $\varangle)$ \\ Department of Electrical and Computer Engineering, University of Florida, Gainesville, FL 32611, USA \\ Received: 12 December 2009 / Revised: 19 January 2010 / Accepted: 20 January 2010 \\ (C) The Author(s) 2010. This article is published with open access at Springerlink.com
}

\begin{abstract}
The band structures of strained graphene nanoribbons (GNRs) are examined using a tight-binding Hamiltonian that is directly related to the type and magnitude of strain. Compared to a two-dimensional graphene whose band gap remains close to zero even if a large strain is applied, the band gap of a graphene nanoribbon (GNR) is sensitive to both uniaxial and shear strains. The effect of strain on the electronic structure of a GNR depends strongly on its edge shape and structural indices. For an armchair GNR, a weak uniaxial strain changes the band gap in a linear fashion, whereas a large strain results in periodic oscillation of the band gap. On the other hand, shear strain always tends to reduce the band gap. For a zigzag GNR, the effect of strain is to change the spin polarization at the edges of GNR, and thereby modulate the band gap. A simple analytical model, which agrees with the numerical results, is proposed to interpret the response of the band gap to strain in armchair GNRs.
\end{abstract}

\section{KEYWORDS}

Graphene nanoribbons (GNRs), band gap, strain

\section{Introduction}

Strain has been extensively used in the silicon electronics industry to boost device performance and has played an important role for the 90-nm technology node [1]. Graphene is an atomically thin two-dimensional (2-D) material and is therefore structurally more amenable than silicon to external modifications including strain. Graphene can also sustain a much larger strain than silicon. The effect of strain on 2-D graphene has been studied both experimentally and theoretically, including the effects of uniform [2-5] and local strains [6] on the electronic structure, as well as the possibility to achieve quantum Hall states in the absence of an external magnetic field [7]. Two-dimensional graphene does not have a band gap, and the band gap remains close to zero even if a strain as large as $20 \%$ is applied. A band gap can be created by patterning the 2-D graphene into a nanometer-wide graphene nanoribbon (GNR); this has been predicted theoretically [8-10] and realized experimentally [11-13]. GNRs present interesting transport properties where, for example, disorder such as imperfect edges, can play an important role [14]. Moreover, strain could be a useful way to further tailor the electronic properties of GNRs. Based on density functional theory (DFT), the effect of uniaxial strain on the electronic properties of GNRs has been studied $[15,16]$. These studies revealed the potential of uniaxial strain as a way of tuning the electronic properties of GNRs. The underlying physics of strain effects on the band gap of GNRs, however, is buried in DFT simulations and is not fully understood.

In this work, a systematic study of the effect of both

Address correspondence to guoj@ufl.edu 
uniaxial and shear strain on the band gap of GNRs is performed using a tight-binding Hamiltonian that is directly related to the magnitude and type of strain. An analytical model is developed to describe the dependence of bandgap on strain in GNRs. The work provides explicit relationships between the bandgap and strain in GNRs, which enables a simple and detailed physical understanding. It is observed that the band gap of a GNR is much more sensitive to strain than 2-D graphene and strongly depends on its edge shape and structural indices. For zigzag GNRs (ZGNRs), uniaxial strain and shear strain modulate the spin density at the GNR edges thereby altering the band gap. For armchair GNRs (AGNRs), uniaxial strain and shear strain result in qualitatively different dependences of the band gap on strain. The effect of strain on the band gap is qualitatively different for AGNRs with different structural indices. The effects of edge bond relaxation [9] and third nearest neighbor coupling [17] modify the quantitative dependence of the band gap on strain.

\section{Approach}

The band structures of the modeled GNRs are calculated by using a tight-binding model, whose binding parameters have been parameterized by ab initio calculations in previous studies of GNR band structures in the absence of strain $[9,17]$. For AGNRs, modeling

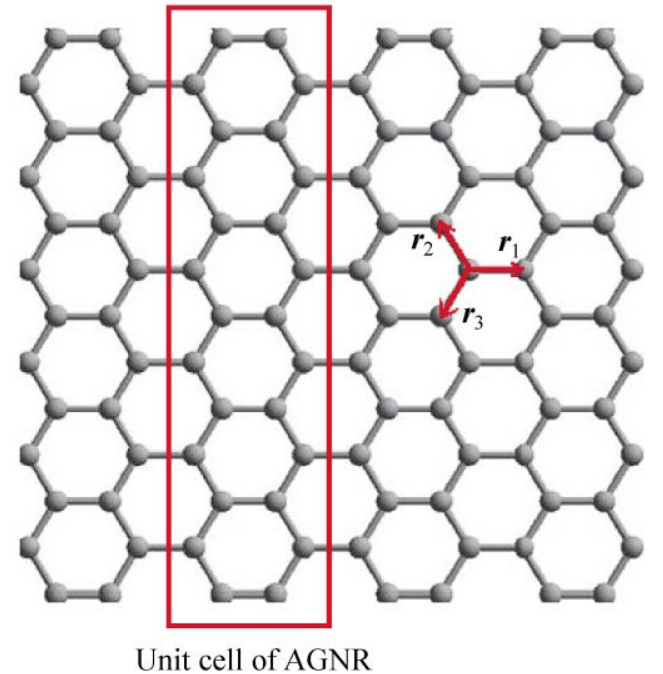

(a) the edge bond relaxation and the third nearest neighbor coupling are necessary to treat the edge effects and describe all semiconducting band structures [13], as predicted by the $a b$ initio calculations $[9,10,17]$. For ZGNRs, inclusion of a Hubbard term in the Hamiltonian is needed to describe the edge spin polarization and opening of the band gap [18]. The binding parameters in the presence of strain are modified according to the Harrison binding parameter relation. This approach has been used and validated before in the study of strain effects on carbon nanotubes [19].

As shown in Fig. 1(a), the unstrained bond vectors for an AGNR are given by

$$
\begin{aligned}
& r_{1}=a_{0} \hat{x} \\
& r_{2}=\frac{1}{2} a_{0} \hat{x}+\frac{\sqrt{3}}{2} a_{0} \hat{y} \\
& r_{3}=\frac{1}{2} a_{0} \hat{x}-\frac{\sqrt{3}}{2} a_{0} \hat{y}
\end{aligned}
$$

where we set $\hat{x}$ as the transport direction of the GNR. The application of a uniaxial or shear strain causes the following changes:

$$
\begin{aligned}
& r_{i x} \rightarrow(1+\sigma) r_{i x} \\
& r_{i y} \rightarrow(1+v \sigma) r_{i y} \\
& r_{i x} \rightarrow r_{i x}+\gamma r_{i y} \quad \text { (Shiaxial strain) }
\end{aligned}
$$

where $i=1,2,3$ and $r_{i x}, r_{i y}$ are the $x$ and $y$ components

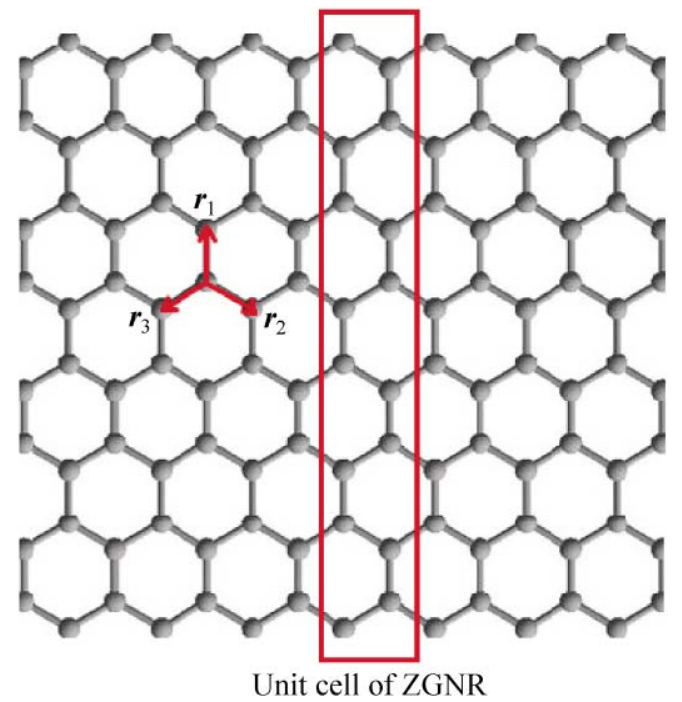

(b)

Figure 1 (a) The unit cell of AGNR (b) unit cell of ZGNR. In each figure, $\boldsymbol{r}_{1}, \boldsymbol{r}_{2}, \boldsymbol{r}_{3}$ are the bond vectors, and the transport direction of the ribbon is set as the $x$ direction 
of $r_{i} . \sigma$ represents the uniaxial strain along the $x$ direction, $v \approx 0.165$ is the Poisson ratio [20], and $\gamma$ is the shear strain. Here we focus on the simple case of uniform strain, while in practice the deformation of graphene may include long range and regular ripple patterns. A tight-binding Hamiltonian as parameterized by Gunlycke and White [21], which includes the treatment of the edge bond relaxation and the third nearest neighbor coupling, is used to compute the band structure of the AGNR. In the presence of strain each binding parameter is scaled by a dimensionless factor $\xi=\left(\frac{r_{0}}{r}\right)^{2}$, where $r_{0}$ the unstrained bond length, and $r$ is the bond length in the presence of strain.

The bond lengths of the zigzag GNR, as shown in Fig. 1(b), are modified in a similar manner to those of the AGNR in the presence of strain. Due to the existence of localized edge states in ZGNRs, the spin polarized interaction should be included in the Hamiltonian of the system, which can be generally described as [22]

$$
H=\sum_{\langle i, j\rangle, \sigma} t_{i j} c_{i \sigma}^{+} c_{j \sigma}+U \sum_{i, \sigma}\left(\left\langle n_{i,-\sigma}\right\rangle-\frac{1}{2}\right) n_{i, \sigma}
$$

where $c_{i \sigma}^{+}, c_{j \sigma}$ and $n_{i \sigma}$ are creation, annihilation, and number operators, respectively, for an electron of spin $\sigma$ in the $\pi$-orbital centered on the $i$-th $\mathrm{C}$ atom in the ribbon. $\langle i, j\rangle$ denotes the set of all nearest neighbors, $t_{i j}$ is the corresponding nearest neighbor hopping parameter and $U$ describes the strength of the spindependent field. $\left\langle n_{i, \sigma}\right\rangle$ is the average electron density with spin $\sigma$ at the location of $i$-th $\mathrm{C}$ atom, and can be calculated self-consistently from equilibrium carrier statistics. The Hamiltonian described by Eq. (6) is in fact equivalent to the Hartree-Fock approximation applied to the Hubbard model [23]. This was first studied by Fujita et al. in their paper on edge states in ZGNRs [24].

\section{Results and discussion}

\subsection{Armchair GNRs}

We first consider the case of uniaxial strain. The band structure $E_{n}(k)$ is calculated and the band gap is obtained by finding the minimum of $2\left|E_{n}(k)\right|$ for all the band indices $n$ and wave vectors $k$. For the purpose of comparison, we first neglect the effect of edge bond relaxation and third nearest neighbor coupling. In this simple case, the band structure of a strained AGNR with an index of $n$ is similar to that of a strained zigzag single-wall nanotube (SWNT) with an index of $n+1$ [17], except for the lack of valley degeneracy. As shown in Fig. 2(a), the band gap scales linearly with the magnitude of the strain over a certain range, and repeats itself periodically as the strain is further increased. The effect of strain on band gap is significant and qualitatively different for AGNRs with $n+1=3 q, 3 q+1$, and $3 q+2$. For the $n=23 \operatorname{AGNR}(3 q)$, small tensile strains increase the band gap, with only $5 \%$ uniaxial strain leading to the opening of a band gap of about $0.4 \mathrm{eV}$. Small tensile strains also increase the band gap of the $n=24$ AGNR $(3 q+1)$, but decrease the band gap of the $n=25$ AGNR $(3 q+2)$.

In the presence of edge bond relaxation and the third nearest neighbor coupling, the band gap is non-zero in the absence of strain for any AGNR. Figure 2(b) shows plots of the band gap of an AGNR under uniaxial strain when the effects of edge bond relaxation and third nearest neighbor coupling are included. The qualitative features of the relationship between band gap and strain do not change, but the quantitative value of the band gap is perturbed. Furthermore, the maximum achievable band gap in the presence of compressive strain is smaller than that in the presence of tensile strain.

In order to obtain a simple relation between strain and the band gap of an AGNR, we can calculate the lowest order contribution of strain to band structures. The eigenenergies of an AGNR at $k=0$ can be written as (see Appendix)

$$
\begin{aligned}
E(p)= & t_{0}\left(1+\alpha+2(1+\beta) \cos \frac{p \pi}{n+1}\right) \\
& +t_{3 n}\left(1+\alpha+2(1+\beta) \cos \frac{2 p \pi}{n+1}\right) \\
& +\frac{4\left(t_{3 n}(1+\beta)+\Delta t_{\mathrm{e}}\right)}{n+1} \sin ^{2} \frac{p \pi}{n+1}
\end{aligned}
$$

where

$$
\alpha=-2 \sigma+3 \sigma^{2}
$$

$$
\beta=-\frac{(1-3 v) \sigma}{2}+\frac{3}{4} \gamma^{2}+\frac{1}{4}(1-3 v)^{2} \sigma^{2}
$$




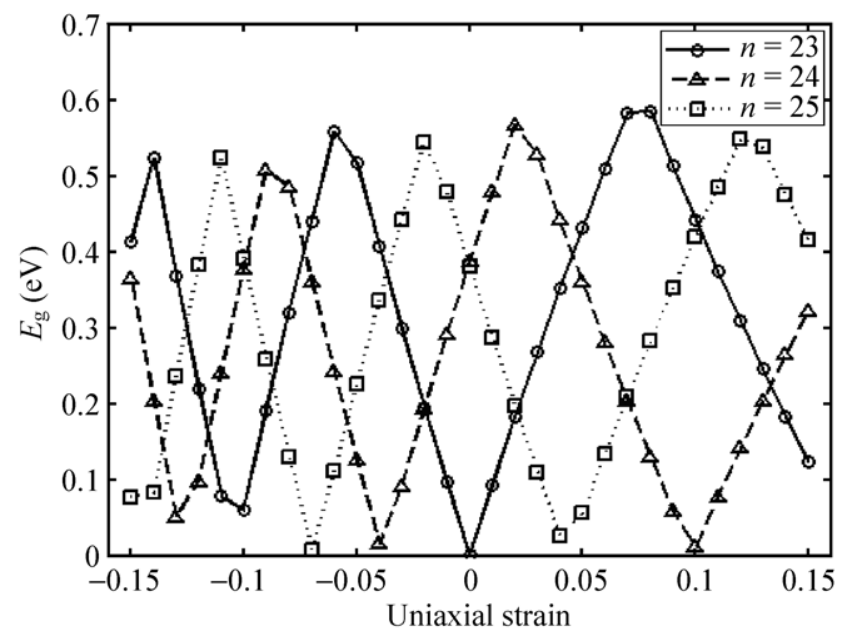

(a)

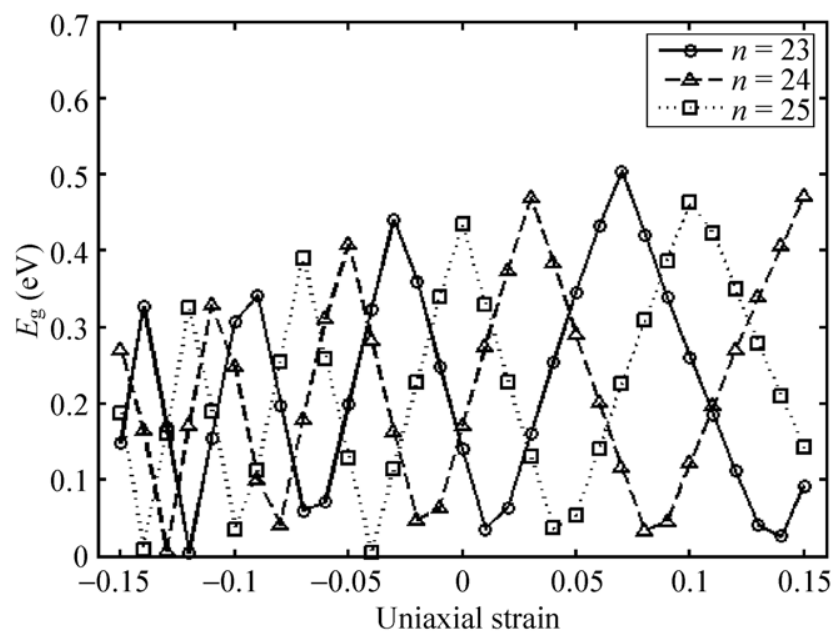

(b)

Figure 2 Plot of band gap versus uniaxial strain for AGNR; $n=$ 23, 24, and 25 correspond to AGNRs with $n+1=3 q, 3 q+1$, and $3 q+2$, respectively. (a) is the simple case neglecting edge bond relaxation and third nearest neighbor coupling effects and (b) includes these two effects. In both figures, the plots of band gap versus strain show similar periodic patterns. Locally, the band gap changes linearly when increasing (decreasing) the magnitude of the strain

Here, $p$ is the band index running from 1 to $n, t_{0}$ is the nearest neighbor hopping integral in the absence of strain, $\sigma$ and $\gamma$ are the magnitudes of uniaxial strain and shear strain, respectively, $v$ is the Poisson ratio, $t_{3 n}$ is the third nearest neighbor coupling strength and $\Delta t_{\mathrm{e}}$ is the correction due to edge bond relaxation. In Eq. (4), the first term corresponds to the band energy neglecting edge bond relaxation and third nearest neighbor coupling, whilst the second and third terms account for these two effects. The quantities $\alpha$ and $\beta$ are the corrections due to strain. Equation (4) gives $n$ of the $2 n$ eigenenergies of the system. The other $n$ eigenenergies, due to symmetry, are just the opposites of Eq. (4). Thus, the band gap can be calculated as

$$
E_{\mathrm{g}}=\min _{P=1,2 \ldots n}|2 E(p)|
$$

From Eq. (4), it is observed that there is no first order contribution to the band gap from shear strain. Also, because $t_{3 n}$ and $\Delta t_{\mathrm{e}}$ are relatively small compared to $t_{0}$, we then preserve only the first term of Eq. (4), which is the dominating factor in the qualitative dependence of the band gap on strain,

$$
E_{\mathrm{g}} \approx 2\left|t_{0}\right| \min _{P=1,2 \ldots n}\left|\left(1+\alpha+2(1+\beta) \cos \frac{p \pi}{n+1}\right)\right|
$$

To the first order of uniaxial strain, Eq. (7) can be further approximated as

$$
\begin{gathered}
E_{g} \approx \min _{p=1,2 \ldots n}\left(-2 \sqrt{3} \pi t_{0}\right)\left|\frac{p}{n+1}-p_{0}\right| \\
p_{0}=\frac{2}{3}-\frac{\sqrt{3}}{2 \pi}(1+v) \sigma
\end{gathered}
$$

where

Equation (8) implies that the band gap is proportional to the shortest distance between $p_{0}$ and $\frac{p}{n+1}$, the quantization grids in the width direction of the GNR. This is depicted in Fig. 3. It is observed that when $n+1=3 q$, the shortest distance of $p_{0}$ to the grids is zero, and for $n+1=3 q+1$ or $3 q+2$, the shortest distance of $p_{0}$ to the grids is $\frac{1}{3(n+1)}$. This explains the features of Fig. 2, as for all three cases, small tensile strains shift the position of $p_{0}$ to the negative direction, which results in the minimum distance of $p_{0}$ to the grids being increased for $n+1=3 q$ or $3 q+1$, and decreased for $n+1=3 q+2$. The behavior in compressive strain can be explained similarly. Figure 3 also provides a qualitative explanation of the periodic oscillation of the band gap as the strain increases. Further shifting the position of $p_{0}$ results in a periodic repetition of that minimum distance, which gives the periodic relationship between band gap and strain. Furthermore, the maximum achievable band gap is proportional to 


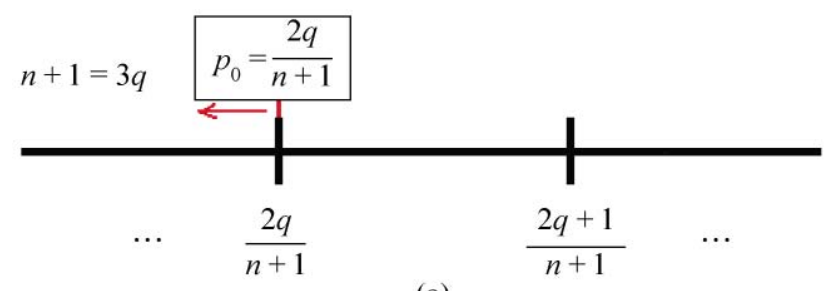

(a)

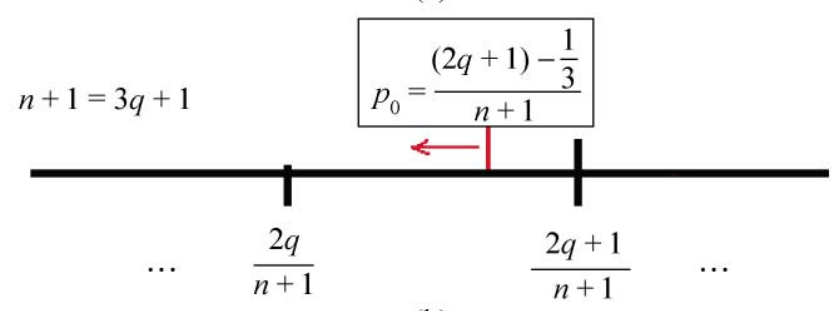

(b)

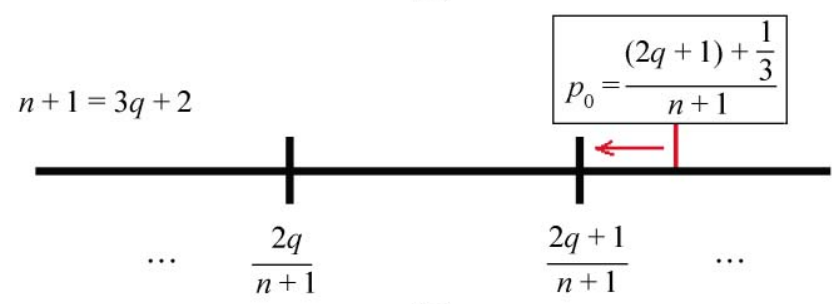

(c)

Figure 3 Visualization of the position of $p_{0}$ in Eq. (8) relative to the quantization grids, $p /(n+1)$, where $p=1$ to $n$. The distance of $p_{0}$ to the nearest grid points is different for AGNRs with different indices. For $n+1=3 q$, it is 0 ; for $n+1=3 q+1$ or $3 q+2$, it is one third of the grid space, $1 /[3(n+1)]$. As indicated by the red arrows, tensile strain shifts $p_{0}$ to the negative direction

half the grid space, $1 /[2(n+1)]$. For an AGNR with $n=3 q$ or $3 q+1$, this corresponds to an increase of about $50 \%$ in the band gap compared to the unstrained case. The explanation of band gap oscillation under strain is similar to previous studies of strain effects on nanotubes, which attributed the change in band gap to the shifting of the Fermi point under strain $[19,25]$.

Plots of the dependence of band gap on both strain and ribbon width are shown in Fig. 4 . The simulated range of uniaxial strain is from $-15 \%$ to $15 \%$, and the width from 2 to $10 \mathrm{~nm}$. The periodic oscillations of the band gap as a function of the magnitude of uniaxial strain and the qualitative difference between $3 q, 3 q+1$, and $3 q+2$ groups are observed for the whole range of simulation parameters. In general, increasing the width of the ribbon reduces the maximum achievable

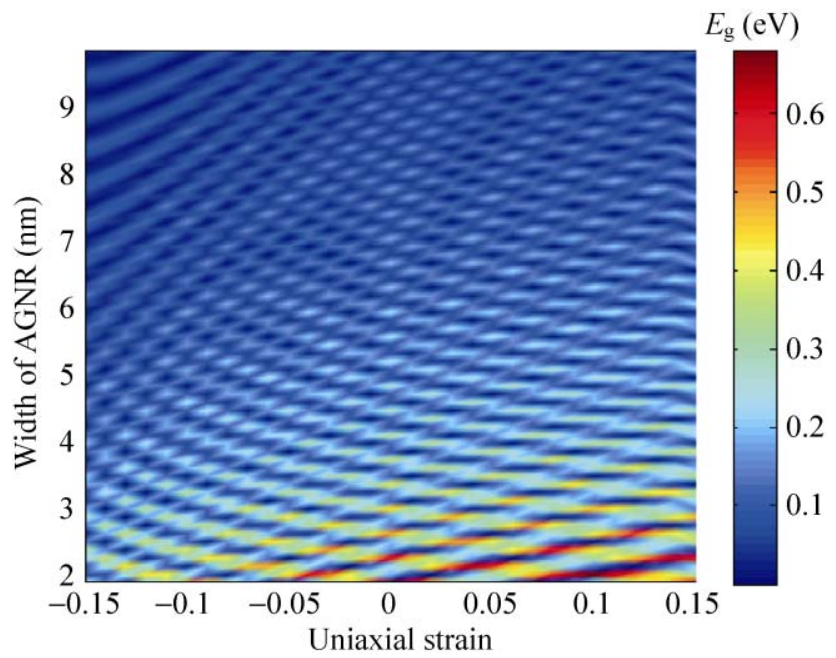

Figure 4 Plots of band gap versus uniaxial strain for AGNRs with different widths, with the effect of edge bond relaxation and third nearest neighbor coupling included. The width of the AGNR varies from 2 to $10 \mathrm{~nm}$. Generally, the band gaps still show a periodic dependence on the magnitude of the strain, and are roughly inversely proportional to the width of the ribbons

band gap, due to the weaker confinement in the width direction.

The effect of shear strain on the band gap of an AGNR is qualitatively different from that of uniaxial strain, as shown in Fig. 5. As Eqs. (4) and (5) indicate, there is no first order contribution from shear strain to the band gap, so the dependence of the band gap on shear strain is due to the second and higher order

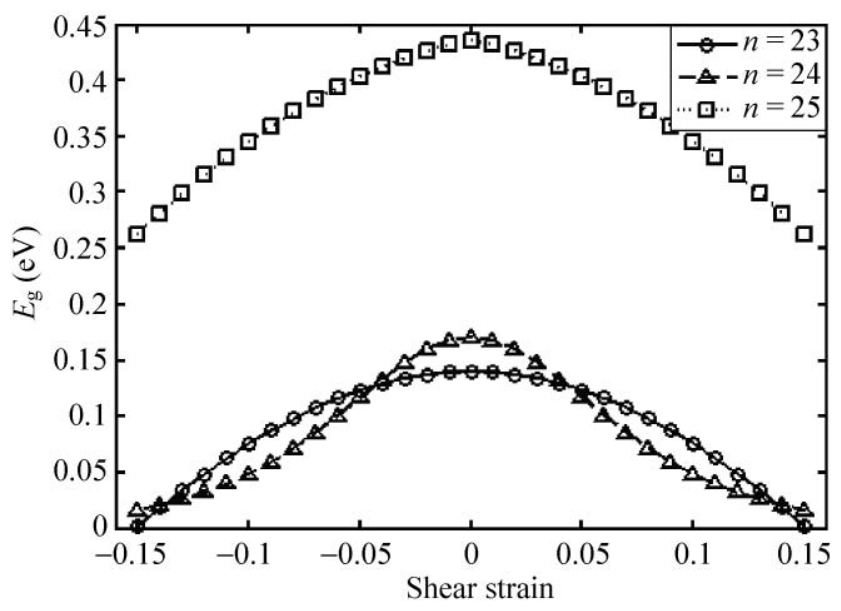

Figure 5 Plots of band gap versus shear strain for AGNRs with edge bond relaxation and third nearest neighbor effect included. In this case, shear strain always tends to reduce the band gap 
perturbation effects. In the presence of edge bond relaxation and third nearest neighbor coupling, shear strain always reduces the band gap regardless of the structural indices of the AGNR.

\subsection{Zigzag GNRs}

The band gap of a ZGNR originates from a totally different mechanism from that for an AGNR, as indicated by Eq. (3). When the spin interaction is included, the band separation at the zone boundary can be approximated as [18]

$$
\Delta E=U\left(n_{1, \uparrow}-n_{1, \downarrow}\right)
$$

The actual band gap is proportional to, but smaller than, $\Delta E$. In Fig. 6, we plot the band structure of a ZGNR with $n=16$. The solid blue lines represent the unstrained band structure while the dashed red lines correspond to the band structure under $15 \%$ uniaxial strain. Obviously, this tensile strain opens up the band gap. The dependence of the band gap of the ZGNR on uniaxial strain is shown in Fig. 7(a). In contrast to AGNRs, the band gap of a ZGNR increases as tensile strain is applied and decreases as compressive strain is applied, regardless of its structural index. A plot of the normalized band gap $E_{\mathrm{g}} / E_{\mathrm{g} 0}$ versus strain is shown

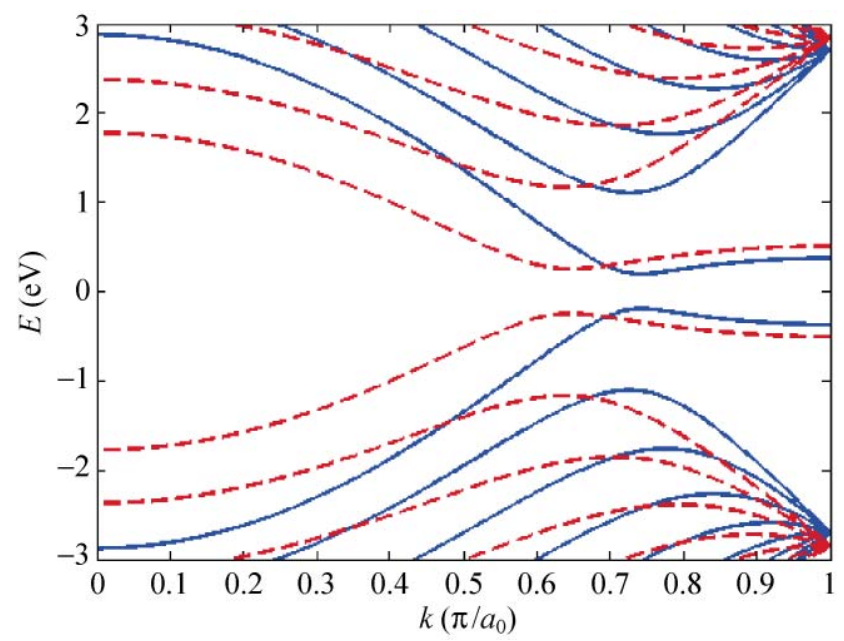

Figure 6 Band structure of a ZGNR with $n=10$. The solid blue lines are the case without strain, and the dashed red lines are the case with $15 \%$ uniaxial strain. In each case, the up spin and down spin band structures are degenerate. It is obvious that the tensile strain increases the band gap in the inset of Fig. 7(a), and is approximately the same for ZGNRs with different widths. Fitting the curve gives the empirical relation,

$$
\frac{E_{g}}{E_{g 0}}=\sigma^{2}+1.6 \sigma+1
$$

where $E_{\mathrm{g} 0}$ is the unstrained band gap. To explain the effect of strain on band gap, we calculated the edge spin polarization for various ZGNRs under uniaxial strain. As shown in Fig. 7(b), positive (negative) uniaxial strain always tend to increase (decrease) the edge spin polarization. As indicated by Eq. (9), stronger spin polarization will induce a larger band separation, which is roughly proportional to the band gap. This justifies the monotonic feature of the plot of band gap versus strain in Fig. 7 .

The reason why tensile strain will increase edge spin polarization can be explained by the analytical model proposed by Fujita [24]. For edge states, the corresponding charge density is proportional to $[2 \cos (k / 2)]^{2 m}$ at each non-nodal site of the $m$ th zigzag chain from the edge. So $2 \cos (k / 2)$ represent the "damping length" of the edge states. If a strain is applied, due to the distortions of bond vector and bond parameter, this damping factor should be modified as $2 \frac{t_{2}}{t_{1}} \cos (k / 2)$, where $t_{1}, t_{2}$ are bond parameters related to the bond vectors $\boldsymbol{r}_{1}, \boldsymbol{r}_{2}$ in Fig. 1(b). For tensile strain $(\sigma>0),\left|t_{2} / t_{1}\right|<1$, the damping of edge states becomes much quicker, which results in more localized edge states. Due to electronelectron interaction, this will increase the spin polarization at edge sites, thereby increasing the band gap of the system. A similar argument applies in the case of compressive strain $(\sigma<0)$.

We also calculated the effect of shear strain on the band gap of a ZGNR, as shown in Fig. 8 (a). Compared to the case of uniaxial strain, the change in band gap is relatively small, and shear strain always tends to reduce the band gap. These features can also be explained by Fujita's model [24]. We find that, under shear strain, the damping factor should be modified as $2 \cos \left(\frac{k}{2}\right) \sqrt{1+\frac{3}{4} \gamma^{2} \tan ^{2}\left(\frac{k}{2}\right)}$, where $\gamma$ 


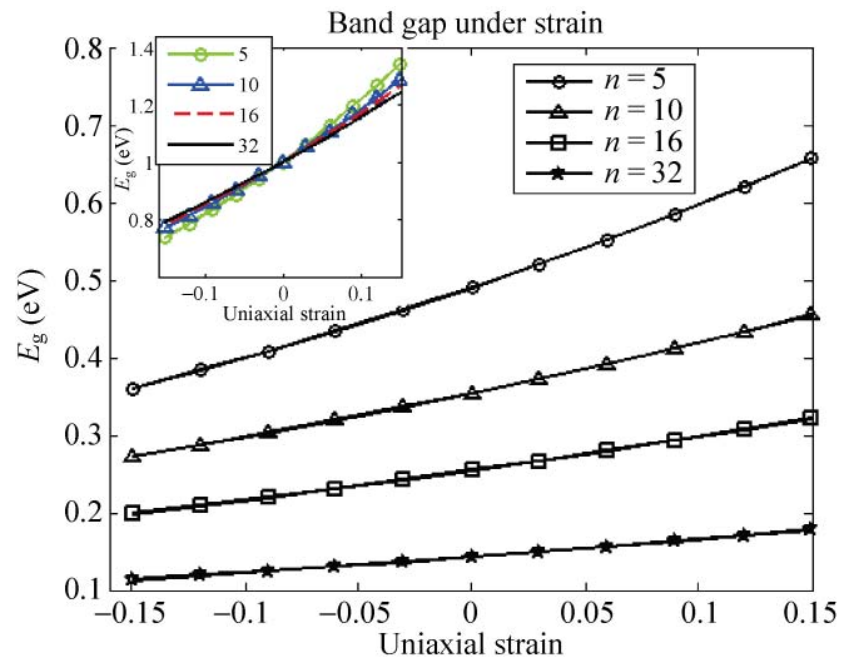

(a)

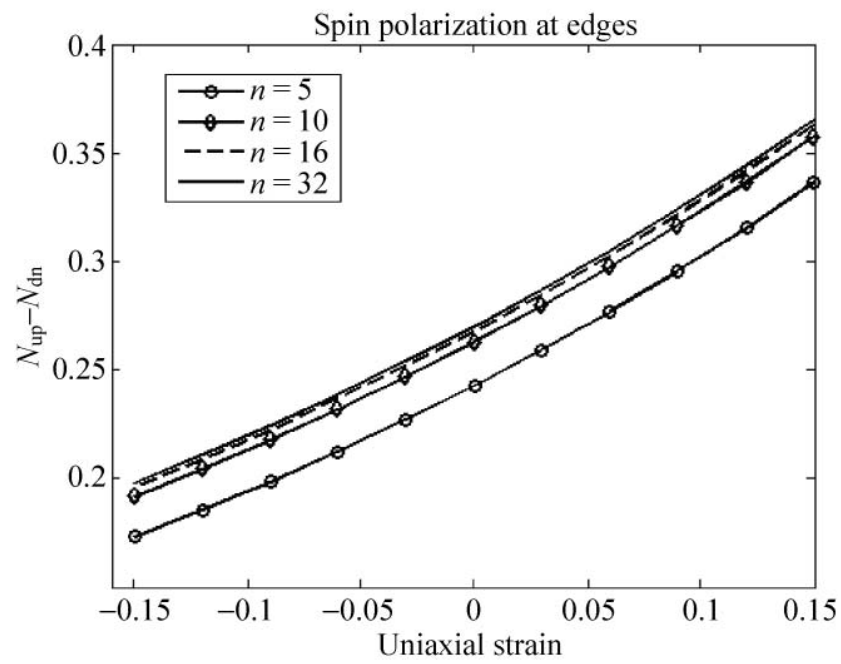

(b)

Figure 7 (a) Band gap of a uniaxially strained ZGNR with different widths (indicated by the number of zigzag chains in the transverse direction); inset is a plot of the normalized band gap versus strain, in which ZGNRs with different widths show a similar linear dependence. (b) The spin polarization (up spin density $\left(N_{\text {up }}\right)$ minus down spin density $\left.\left(N_{\mathrm{dn}}\right)\right)$ at the edges of the ZGNR. Tensile (compressive) strain increases (decreases) spin polarization

is the magnitude of the shear strain. Because $\sqrt{1+\frac{3}{4} \gamma^{2} \tan ^{2}\left(\frac{k}{2}\right)} \geqslant 1$, under shear strain the damping of edge states is slower than without strain. So the edge states are less localized, thus decreasing the spin polarization at edge sites, and therefore reducing the band gap. This analysis is confirmed by Fig. 8(b), in which shear strain always reduce the spin polarization at the edges.

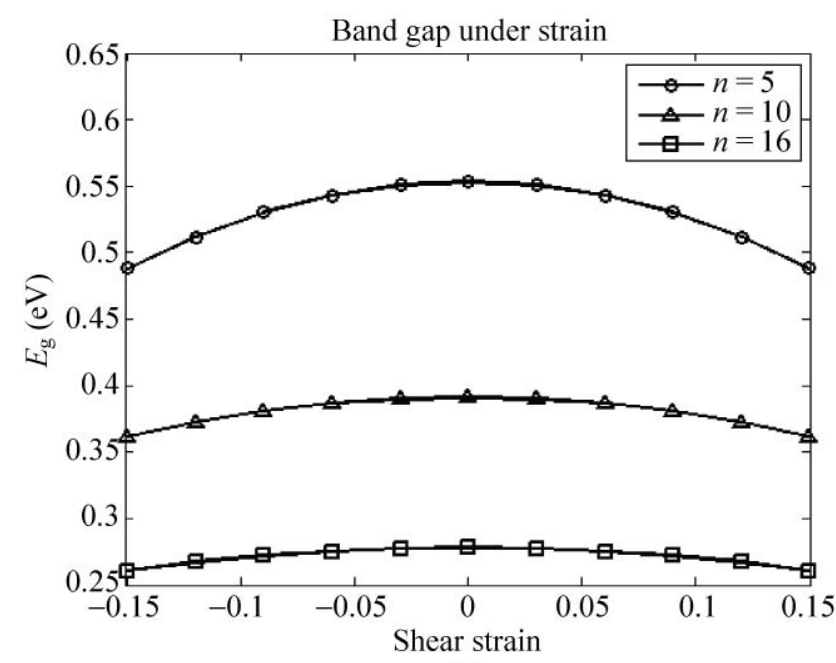

(a)

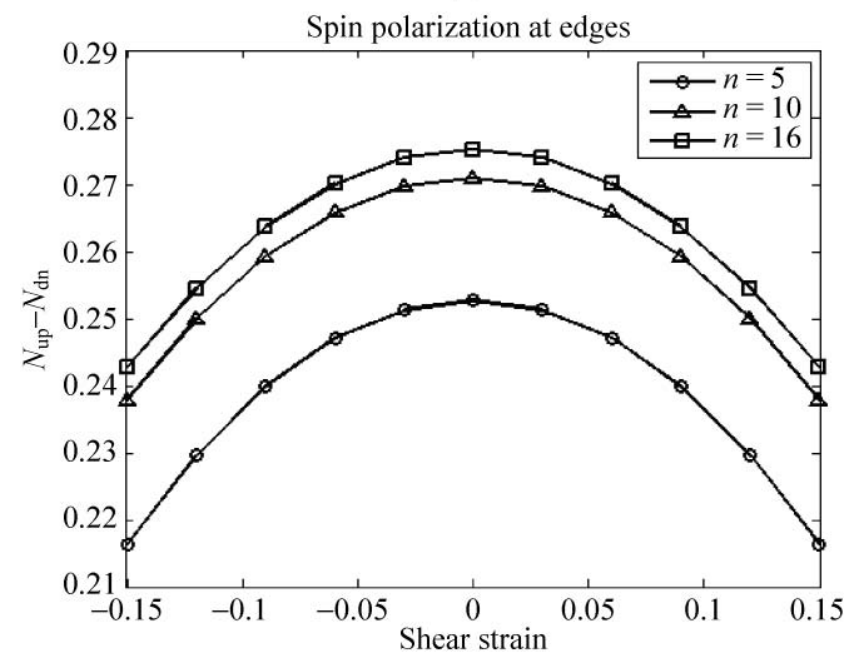

(b)

Figure 8 (a) The band gap of shear strained ZGNR with different widths (indicated by the number of zigzag chains in the transverse direction). (b) The spin polarization (up spin density $\left(N_{\text {up }}\right)$ minus down spin density $\left(N_{\mathrm{dn}}\right)$ ) at the edges of the ZGNR. Shear strain always tends to decrease spin polarization at the edges

\section{Conclusions}

We have explored the effect of strain on the band gap of GNRs. Two types of strain (uniaxial strain and shear strain) and two types of GNRs (AGNRs and ZGNRs) have been studied. The effect of strain is modeled as a modification to the tight-binding nearest neighbor hopping integral. It is found that for an AGNR, uniaxial strain linearly shifts the band gap in a way which periodically repeats itself as the magnitude of the strain is increased. Shear strain makes no obvious 
contribution to the opening up of the band gap and, in all cases, it tends to reduce the band gap. We explained these observations by proposing a perturbation model and it reproduced well the results of the numerical calculations. For a ZGNR, we found that strain changes the spin polarization at edge sites of the nanoribbon, thus further affecting the band gap. Tensile strain increases the band gap while compressive and shear strain reduce the band gap. These results indicate that, the band gap of a GNR is sensitive to magnitude of the strain to which it is subjected. By applying moderate strains, the electronic properties of GNRs can be readily engineered.

\section{Acknowledgements}

This work was supported by Office of Naval Research (ONR) and the National Science Foundation (NSF).

Open Access: This article is distributed under the terms of the Creative Commons Attribution Noncommercial License which permits any noncommercial use, distribution, and reproduction in any medium, provided the original author(s) and source are credited.

\section{References}

[1] International technology road map for semiconductors. http://www.itrs.net/

[2] Pereira, V. M.; Castro Neto, A. H.; Peres, N. M. R. Tightbinding approach to uniaxial strain in graphene. Phys. Rev. B 2009, 80, 045401.

[3] Gui, G.; Li, J.; Zhong, J. X. Band structure engineering of graphene by strain: First-principles calculations. Phys. Rev. B 2008, 78, 075435.

[4] Farjam, M.; Rafii-Tabar, H. Comment on "Band structure engineering of graphene by strain: First-principles calculations". Phys. Rev. B 2009, 80, 167401.

[5] Gui, G.; Li, J.; Zhong, J. X. Reply to "Comment on 'Band structure engineering of graphene by strain: First-principles calculations"”. Phys. Rev. B 2009, 80, 167402.

[6] Pereira, V. M.; Castro Neto, A. H. Strain engineering of graphene's electronic structure. Phys. Rev. Lett. 2009, 103, 046801.

[7] Guinea, F.; Katsnelson, M. I.; Geim, A. K. Energy gaps and a zero-field quantum Hall effect in graphene by strain engineering. Nat. Phys. 2009, 6, 30-33.

[8] Eazwa, M. Peculiar width dependence of the electronic properties of carbon nanoribbons. Phys. Rev. B 2006, 73, 045432.

[9] Son, Y. W.; Cohen, M. L.; Louie, S. G. Energy gaps in graphene nanoribbons. Phys. Rev. Lett. 2006, 97, 216803.

[10] Barone, V.; Hod, O.; Scuseria, G. E. Electronic structure and stability of semiconducting graphene nanoribbons. Nano Lett. 2006, 6, 2748-2754.

[11] Han, M. Y.; Ozyilmaz, B.; Zhang, Y. B.; Kim, P. Energy band-gap engineering of graphene nanoribbons. Phys. Rev. Lett. 2007, 98, 206805.

[12] Chen, Z. H.; Lin, Y. M.; Rooks, M. J.; Avouris, P. Graphene nano-ribbon electronics. Physica E 2007, 40, 228-232.

[13] Li, X. L.; Wang, X. R.; Zhang, L.; Lee, S. W.; Dai, H. J. Chemically derived, ultrasmooth graphene nanoribbon semiconductors. Science 2008, 319, 1229-1232.

[14] Cresti, A.; Nemec, N.; Biel, B.; Niebler, G.; Triozon, F.; Cuniberti, G.; Roche, S. Charge transport in disordered graphene-based low dimensional materials. Nano Res. 2008, 1, 361-394.

[15] Sun, L.; Li, Q. X.; Ren, H.; Su, H. B.; Shi, Q. W.; Yang, J. L. Strain effect on electronic structures of graphene nanoribbons: A first-principles study. J. Chem. Phys. 2008, 129, 074704.

[16] Hod, O.; Scuseria, G. E. Electromechanical properties of suspended graphene nanoribbons. Nano Lett. 2009, 9, 2619-2622.

[17] White, C. T.; Li, J.; Gunlycke, D.; Mintmire, J. W. Hidden one-electron interactions in carbon nanotubes revealed in graphene nanostrips. Nano Lett. 2007, 7, 825-830.

[18] Gunlycke, D.; Areshkin, D. A.; Li, J. W.; Mintmire, J. W.; White, C. T. Graphene nanostrip digital memory device. Nano Lett. 2007, 7, 3608-3611.

[19] Yang, L.; Han, J. Electronic structure of deformed carbon nanotubes. Phys. Rev. Lett. 2000, 85, 154-157.

[20] Blakslee, O. L.; Proctor, D. G.; Seldin, E. J.; Spence, G. B.; Weng, T. Elastic constants of compression-annealed pyrolytic graphite. J. Appl. Phys. 1970, 41, 3373-3382.

[21] Gunlycke, D.; White, C. T. Tight-binding energy dispersions of armchair-edge graphene nanostrips. Phys. Rev. B 2008, $77,115116$.

[22] Guo, J.; Gunlycke, D.; White, C. T. Field effect on spinpolarized transport in graphene nanoribbons. Appl. Phys. Lett. 2008, 92, 163109.

[23] Hubbard, J. Electron correlations in narrow energy bands. Proc. R. Soc. Lond. A 1963, 276, 238-257. 
[24] Fujita, M.; Wakabayashi, K.; Nakada, K. Peculiar localized state at zigzag graphite edge. J. Phys. Soc. Jpn. 1996, 65, 1920-1923.

[25] Nagapriya, K. S.; Berber, S.; Cohen-Karni, T.; Segev, L.; Srur-Lavi, O.; Tománek, D.; Joselevich, E. Origin of torsioninduced conductance oscillations in carbon nanotubes. Phys. Rev. B 2008, 78, 165417.

\section{Appendix}

\section{Derivation of Eq. (4)}

For an AGNR, in the tight-binding model the band gap always occurs at $k=0$ due to the symmetry of the Hamiltonian. At $k=0$, the tight-binding Hamiltonian reduces to a two leg ladder lattice system [9], as shown in Fig. A.1.
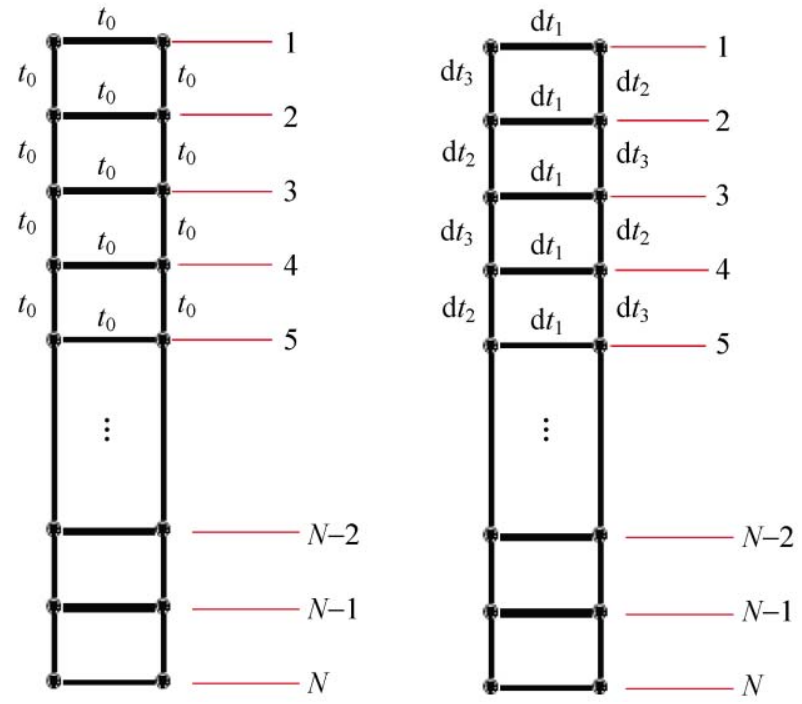

Figure A.1 The $k=0$ Hamiltonian used in the Appendix: (a) the unstrained Hamiltonian; (b) the strain-induced perturbation Hamiltonian

$$
H_{0}=\left(\begin{array}{cc}
T & t_{0} I \\
t_{0} I & T
\end{array}\right)
$$

where $\quad T=\left(\begin{array}{cccc}0 & t_{0} & & \\ t_{0} & 0 & t_{0} & \\ & t_{0} & \ldots & t_{0} \\ & & t_{0} & 0\end{array}\right)$ corresponds to the left (right) leg of the ladder.

The eigenstates and eigenenergies of $H_{0}$ are

$$
H_{0}\left|E_{p}\right\rangle=E_{p}\left|E_{p}\right\rangle, \quad E_{p}=t_{0}\left(1+2 \cos \frac{p \pi}{n+1}\right), \quad p=1,2 \ldots n
$$

where $|E\rangle=\left(\begin{array}{l}\psi_{p} \\ \psi_{p}\end{array}\right), \quad \psi_{p}=\left(\begin{array}{c}\sin \frac{p \pi}{n+1} \\ \sin \frac{2 p \pi}{n+1} \\ \ldots \\ \sin \frac{n p \pi}{n+1}\end{array}\right)$

Equation (A.2) describes $n$ of the $2 n$ eigenstates we would like to discuss. The other $n$ eigenstates, due to symmetry, just have the opposite eigenenergies, $-E_{p}$, $p=1,2 \ldots n$.

The perturbed Hamiltonian and energy due to strain are

$$
\begin{aligned}
& H^{\prime}=\left(\begin{array}{cc}
T_{\mathrm{L}} & \mathrm{d} t_{1} I \\
\mathrm{~d} t_{1} I & T_{\mathrm{R}}
\end{array}\right), \quad T_{\mathrm{L}}=\left(\begin{array}{cccc}
0 & \mathrm{~d} t_{3} & & \\
\mathrm{~d} t_{3} & 0 & \mathrm{~d} t_{2} & \\
& \mathrm{~d} t_{2} & \ldots & \mathrm{d} t_{3} \\
& & \mathrm{~d} t_{3} & 0
\end{array}\right), \\
& T_{\mathrm{R}}=\left(\begin{array}{cccc}
0 & \mathrm{~d} t_{2} & & \\
\mathrm{~d} t_{2} & 0 & \mathrm{~d} t_{3} & \\
& \mathrm{~d} t_{3} & \ldots & \mathrm{d} t_{2} \\
& & \mathrm{~d} t_{2} & 0
\end{array}\right) \\
& \Delta E_{p}=\frac{\left\langle E_{p}\left|H^{\prime}\right| E_{p}\right\rangle}{\left\langle E_{p} \mid E_{p}\right\rangle}=\frac{\left(\begin{array}{ll}
\psi_{p}^{+} & \psi_{p}^{+}
\end{array}\right)\left(\begin{array}{cc}
T_{\mathrm{L}} & \mathrm{d} t_{1} I \\
\mathrm{~d} t_{1} I & T_{\mathrm{R}}
\end{array}\right)\left(\begin{array}{l}
\psi_{p} \\
\psi_{p}
\end{array}\right)}{2 \psi_{p}^{+} \psi_{p}} \\
& =\frac{\psi_{p}^{+}\left(T_{\mathrm{L}}+T_{\mathrm{R}}\right) \psi_{p}+2 \mathrm{~d} t_{1} \psi_{p}^{+} \psi_{p}}{2 \psi_{p}^{+} \psi_{p}}=\mathrm{d} t_{1}+\frac{\psi_{p}^{+}\left(T_{\mathrm{L}}+T_{\mathrm{R}}\right) \psi_{p}}{2 \psi_{p}^{+} \psi_{p}} \\
& =\mathrm{d} t_{1}+2 \frac{\left(\mathrm{d} t_{2}+\mathrm{d} t_{3}\right)}{(N+1)} \sum_{k=1}^{N-1} \sin \frac{k p \pi}{n+1} \sin \frac{(k+1) p \pi}{n+1} \\
& =\mathrm{d} t_{1}+\left(\mathrm{d} t_{2}+\mathrm{d} t_{3}\right) \cos \frac{p \pi}{n+1}
\end{aligned}
$$

Using Eqs. (A.2) and (A.3) we can obtain the expressions for the perturbed bonding parameter, 


$$
\begin{aligned}
\mathrm{d} t_{1}= & t_{0}\left(-2 \sigma+3 \sigma^{2}\right) \\
\mathrm{d} t_{2}= & t_{0}\left(+\frac{\sqrt{3}}{2} \gamma-\frac{(1-3 v) \sigma}{2}+\frac{3}{4} \gamma^{2}+\frac{1}{4}(1-3 v)^{2} \sigma^{2}\right. \\
& \left.-\frac{\sqrt{3}}{2}(1-3 v) \gamma \sigma\right) \\
\mathrm{d} t_{3}= & t_{0}\left(-\frac{\sqrt{3}}{2} \gamma-\frac{(1-3 v) \sigma}{2}+\frac{3}{4} \gamma^{2}+\frac{1}{4}(1-3 v)^{2} \sigma^{2}\right. \\
+ & \left.\frac{\sqrt{3}}{2}(1-3 v) \gamma \sigma\right)
\end{aligned}
$$

Substituting Eq. (A.4) into Eq. (A.3), we have

$$
\begin{aligned}
\Delta E_{p}= & t_{0}\left[\left(-2 \sigma+3 \sigma^{2}\right)+\left((3 v-1) \sigma+\frac{1}{2}(1-3 v)^{2} \sigma^{2}\right.\right. \\
& \left.\left.+\frac{3}{2} \gamma^{2}\right) \cos \frac{p \pi}{n+1}\right]
\end{aligned}
$$

To include the effect of edge distortion and third nearest neighbor coupling, we use a similar method to the above, namely the perturbed Hamiltonian due to the third nearest neighbor coupling and edge bond relaxation,

$$
\begin{aligned}
H_{3 n} & =\left(\begin{array}{cc}
0 & T_{3 n} \\
T_{3 n}^{\prime} & 0
\end{array}\right), \\
T_{3 n} & =\left(\begin{array}{cccccccc}
t_{3 n}^{(1)} & 0 & t_{3 n}^{(2)} & & & & \\
0 & t_{3 n}^{(1)} & 0 & t_{3 n}^{(3)} & & & \\
t_{3 n}^{(3)} & 0 & t_{3 n}^{(1)} & 0 & t_{3 n}^{(2)} & & \\
& t_{3 n}^{(2)} & 0 & t_{3 n}^{(1)} & 0 & \ddots & \\
& & t_{3 n}^{(3)} & 0 & \ddots & \ddots & t_{3 n}^{(3)} \\
& & & \ddots & \ddots & t_{3 n}^{(1)} & 0 \\
& & & & t_{3 n}^{(2)} & 0 & t_{3 n}^{(1)}
\end{array}\right) \\
H_{\text {edge }} & =\left(\begin{array}{cc}
0 & T_{\mathrm{e}} \\
T_{\mathrm{e}}^{\prime} & 0
\end{array}\right), \quad T_{\mathrm{e}}=\left(\begin{array}{ccccc}
\Delta t_{\mathrm{e}} & & & \\
& & 0 & & \\
& & \ddots & \\
& & & 0 & \\
& & & & \Delta t_{\mathrm{e}}
\end{array}\right)
\end{aligned}
$$

where $\Delta t_{\mathrm{e}}$ is the edge bond correction and $t_{3 n}^{(1)}, t_{3 n}^{(2)}$, and $t_{3 n}^{(3)}$ are the third nearest neighbor coupling parameters under strain, which are given by expressions similar to Eq. (A.4)

$$
\begin{aligned}
t_{3 n}^{(1)} & =t_{3 n}\left(1-2 \sigma+3 \sigma^{2}\right) \\
t_{3 n}^{(2)} & =t_{3 n}\left(1+\frac{\sqrt{3}}{2} \gamma-\frac{(1-3 v) \sigma}{2}\right. \\
& \left.+\frac{3}{4} \gamma^{2}+\frac{1}{4}(1-3 v)^{2} \sigma^{2}-\frac{\sqrt{3}}{2}(1-3 v) \gamma \sigma\right) \\
t_{3 n}^{(3)} & =t_{3 n}\left(1-\frac{\sqrt{3}}{2} \gamma-\frac{(1-3 v) \sigma}{2}+\frac{3}{4} \gamma^{2}\right. \\
& \left.+\frac{1}{4}(1-3 v)^{2} \sigma^{2}+\frac{\sqrt{3}}{2}(1-3 v) \gamma \sigma\right)
\end{aligned}
$$

where $t_{3 n}$ is the unstrained third nearest neighbor coupling parameter.

The perturbed energy is given by

$$
\begin{aligned}
\Delta E_{p 3 n}= & \frac{\left\langle E_{p}\left|H_{3 n}\right| E_{p}\right\rangle}{\left\langle E_{p} \mid E_{p}\right\rangle}=\frac{\psi_{p}^{+}\left(T_{3 n}+T_{3 n}^{\prime}\right) \psi_{p}}{n+1} \\
= & t_{3 n}^{(1)}+\left(t_{3 n}^{(2)}+t_{3 n}^{(3)}\right) \cos \frac{2 p \pi}{n+1}+\frac{2\left(t_{3 n}^{(2)}+t_{3 n}^{(3)}\right)}{n+1} \sin ^{2} \frac{p \pi}{N+1} \\
= & t_{3 n}\left(1-2 \sigma+3 \sigma^{2}\right)+2 t_{3 n}\left(1-\frac{(1-3 v) \sigma}{2}+\frac{3}{4} \gamma^{2}\right. \\
& \left.+\frac{1}{4}(1-3 v)^{2} \sigma^{2}\right) \cos \frac{2 p \pi}{n+1}+\frac{4 t_{3 n}}{n+1}\left(1-\frac{(1-3 v) \sigma}{2}\right. \\
& \left.+\frac{3}{4} \gamma^{2}+\frac{1}{4}(1-3 v)^{2} \sigma^{2}\right) \sin ^{2} \frac{p \pi}{n+1} \\
\Delta E_{p \mathrm{e}} & =\frac{\left\langle E_{p}\left|H_{\text {edge }}\right| E_{p}\right\rangle}{\left\langle E_{p} \mid E_{p}\right\rangle}=\frac{2 \psi_{p}^{+} T_{\mathrm{e}} \psi_{p}}{n+1}=\frac{4 \Delta t_{\mathrm{e}}}{n+1} \sin ^{2} \frac{p \pi}{n+1}
\end{aligned}
$$

Adding Eqs. (A.2), (A.5), (A.6), and (A.7) we get

$$
\begin{aligned}
E(p)= & t_{0}\left(1+\alpha+2(1+\beta) \cos \frac{p \pi}{n+1}\right) \\
& +t_{3 n}\left(1+\alpha+2(1+\beta) \cos \frac{2 p \pi}{n+1}\right) \\
& +\frac{4\left(t_{3 n}(1+\beta)+\Delta t_{\mathrm{e}}\right)}{n+1} \sin ^{2} \frac{p \pi}{n+1}
\end{aligned}
$$

where

$$
\begin{aligned}
& \alpha=-2 \sigma+3 \sigma^{2} \\
& \beta=-\frac{(1-3 v) \sigma}{2}+\frac{3}{4} \gamma^{2}+\frac{1}{4}(1-3 v)^{2} \sigma^{2}
\end{aligned}
$$


To see the effectiveness of this analytical approxiby the tight-binding model in Section 2 with those mation, we compared the results calculated numerically using Eqs. (9) to (11), as shown in Fig. A.2.

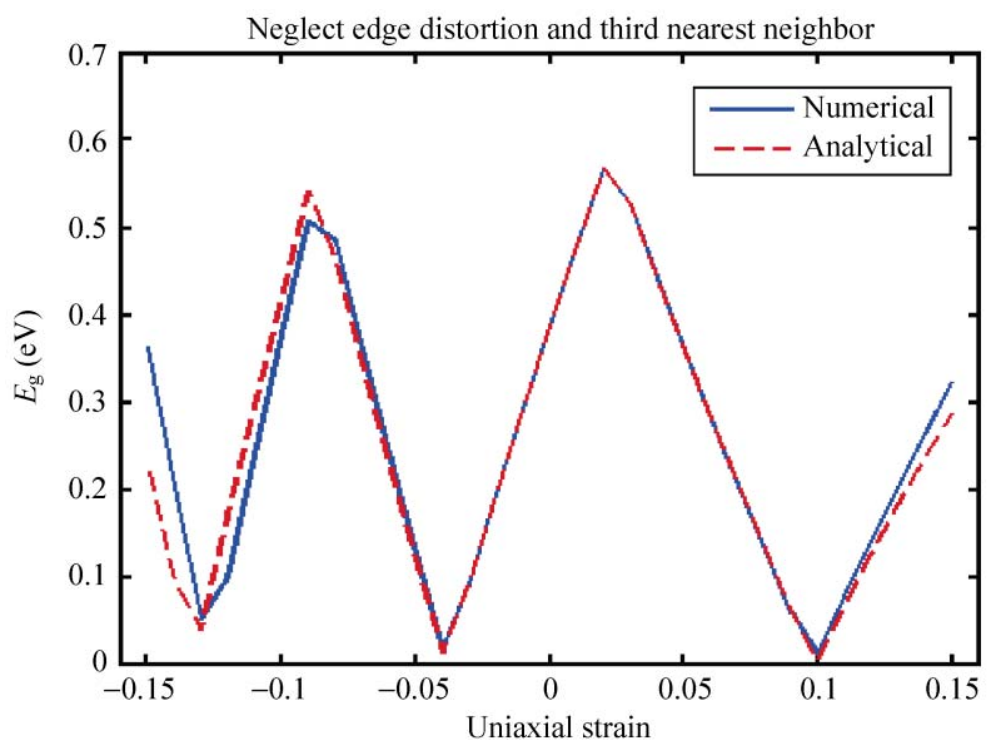

(a)

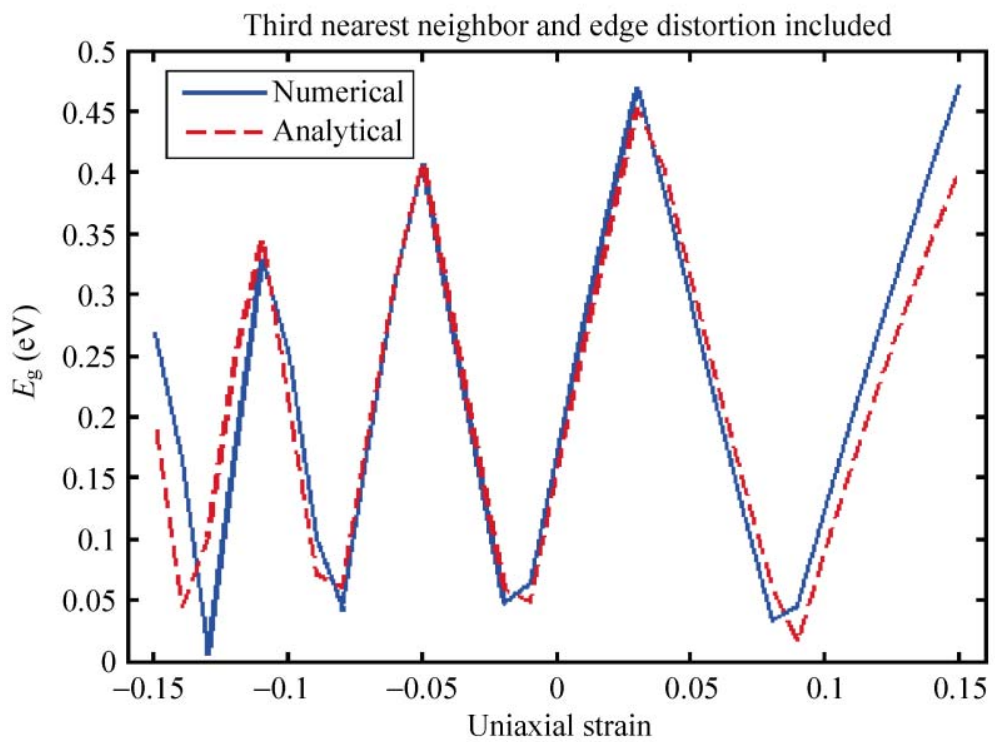

(b)

Figure A.2 Band gap of a strained AGNR with $n=24$, showing a comparison between numerical calculation (solid blue lines) and the analytical model (dashed red lines) developed in this section. In (a) edge distortion and third nearest neighbor coupling are ignored, whilst in (b) these two effects are included. The analytical model agrees well with the numerical simulation results. The deviations between the two treatments in the high strain region may be due to higher order effects 